\title{
Uma proposta, duas experiências: a Fundação Rockefeller e a formação de enfermeiras no Brasil e na Argentina (primeira metade do século $X X)$
}

\author{
One proposal, two experiences: the Rockefeller Foundation and the training of \\ nurses in Brazil and Argentina (first half of the 20th century)
}

\author{
Una propuesta, dos experiencias: la Fundación Rockefeller y la formación de \\ enfermeras en Brasil y Argentina (primera mitad del siglo XX)
}

Ana Paula Korndörfer aninha.korndorfer@gmail.com

Programa de Pós-Graduação em História da Universidade do Vale do Rio dos Sinos, Brasil

(iD) Karina Inés Ramacciotti

karinaramacciotti@gmail.com

Universidad Nacional de Quilmes, Consejo Nacional de Investigaciones Científicas y Técnicas, Argentina

Recepção: 31 Julho 2020

Aprovação: 04 Dezembro 2020

Publicación: 01 Marzo 2021

Cita sugerida: Korndörfer, A. P. y Ramacciotti, K. I. (2021). Uma proposta, duas experiências: a Fundação Rockefeller e a formação de enfermeiras no Brasil e na Argentina (primeira metade do século XX). Descentrada, 5(1), e131. https://doi.org/10.24215/25457284e131
Resumo: Este texto tem como objetivo discutir a atuação da Divisão Internacional de Saúde (DIS) da Fundação Rockefeller (FR), instituição filantrópica norte-americana, na formação de enfermeiras no Brasil e na Argentina na primeira metade do século $\mathrm{XX}$. $\mathrm{O}$ artigo, que traz uma discussão historiográfica e a análise de fontes produzidas, por exemplo, pela própria Fundação, está dividido em três partes. Na primeira seção, expomos algumas concepções da DIS em torno da enfermagem moderna. Nas duas últimas, abordamos a formação de enfermeiras nos dois países em questão, sublinhando semelhanças e diferenças.

Palavras-chave: Fundação Rockefeller, Enfermagem, Formação profissional, Brasil, Argentina.

Abstract: This text aims to discuss the role of the International Health Division (IHD) of the Rockefeller Foundation (RF), a North American philanthropic institution, in the training of nurses in Brazil and Argentina in the first half of the 20th century. The article, which includes a historiographical discussion and analysis of sources produced, for example, by the Foundation itself, is divided into three parts. In the first section, some conceptions of the IHD around modern nursing are shown. In the last two, the training of nurses in the two countries in question is addressed, highlighting similarities and differences.

Keywords: Rockefeller Foundation, Nursing, Professional training, Brazil, Argentina.

Resumen: Este escrito tiene como objetivo discutir la actuación de la División Internacional de Salud (DIS) de la Fundación Rockefeller (FR), institución filantrópica norteamericana, en la formación de enfermeras en Brasil y en Argentina en la primera mitad del siglo XX. El artículo, que traza una discusión historiográfica y un análisis de fuentes producidas por la propia 
Fundación, está dividido en tres partes. En la primera sección, exponemos algunos conceptos de la DIS en torno a la enfermería moderna. En las dos últimas, abordamos la formación de las enfermeras en los dos países en cuestión señalando semejanzas y diferencias.

Palabras clave: Fundación Rockefeller, Enfermería, Formación profesional, Brasil, Argentina.

\section{INTRODUCCIÓN}

No início do século XX, a enfermagem se constitui como uma profissão moderna, majoritariamente exercida pelo sexo feminino. Havia uma preocupação em estimular o ingresso de mulheres na profissão, bem como realizar sua capacitação. Estados nacionais da América do Sul, por exemplo, tiveram atuação importante neste sentido, assim como uma instituição norte-americana, a Fundação Rockefeller. Este texto se propõe a discutir a atuação da Fundação na formação de enfermeiras no Brasil e na Argentina na primeira metade do século XX.

A Fundação Rockefeller (FR), instituição filantrópica norte-americana, é definida, de maneira geral, como uma "organização beneficente, não governamental, que utiliza recursos próprios para financiar atividades de bem-estar social em vários países do mundo” (Faria, 2007, p. 103). Criada em 1913 com o objetivo de incorporar, em uma única organização, instituições pertencentes à família Rockefeller como a General Education Board e a Sanitary Commission for the Eradication of Hookworm Disease, se dedicou a estimular atividades culturais, científicas e sanitárias em diferentes partes do mundo. Entre 1913 e 1951, esteve em funcionamento a Divisão Internacional de Saúde (DIS), ${ }^{1}$ programa cuja missão foi combater a ancilostomíase, a febre amarela e a malária. O possível contágio de algumas enfermidades nos Estados Unidos, a partir da abertura do Canal do Panamá, levou ao financiamento de projetos em diferentes países da América Latina com o objetivo de prevenir e erradicar doenças (Wilkinson, 2000). Durante o mesmo período, a DIS fundou uma série de escolas de saúde pública na América, Europa e Ásia e distribuiu milhares de bolsas de estudo para profissionais da saúde (Farley, 2004; Birn, 2006).

Visando à organização das ações sanitárias fomentadas pela DIS na América Latina, dividiu-se o território em regiões; estas não equivaliam, necessariamente, às fronteiras nacionais. Em 1917, a DIS, que iniciava os trabalhos de combate à ancilostomíase no Brasil, estabeleceu um escritório central no país, localizado em Niterói, no estado do Rio de Janeiro (Korndorfer, 2013). Em 1941, por sua vez, a DIS criou uma nova dependência regional para o Rio da Prata e a Região Andina e que abrangia a zona compreendida por Argentina, Chile, Peru, Equador, Bolívia, Uruguai e Paraguai (Ramacciotti, 2017).

A Fundação Rockefeller desempenhou um importante papel no que se refere à saúde pública, inclusive na América do Sul. Antes da fundação da Organização Mundial da Saúde (OMS), em 1948, a DIS foi a agência de atuação em saúde pública mais importante do mundo. Durante a primeira metade do século XX, nenhuma agência teve tanto alcance ou foi tão ativa na promoção da saúde pública internacional quanto a Fundação Rockefeller (Farley, 2004; Birn, 2006). Entre as décadas de 1920 e 1960, a Fundação ajudou a construir uma extensa rede de instituições científicas que propiciaram a difusão e a consolidação de um modelo de ciência alinhada às ideias norte-americanas. Priorizar a capacitação de indivíduos em detrimento da criação de instituições foi um recurso estratégico para expandir e posicionar os Estados Unidos como principal centro de influência acadêmico e científico, superando a França (Faria e Costa, 2006; Faria, 2007; Cueto, 1994; Gutiérrez Urquijo, 2019).

Este texto, que se propõe a discutir a atuação da Fundação Rockefeller na formação de enfermeiras no Brasil e na Argentina na primeira metade do século XX, está dividido em três seções. ${ }^{2}$ Na primeira parte, 
expomos algumas concepções da DIS em torno da enfermagem moderna. Nas duas últimas, abordaremos a formação de enfermeiras nos dois países em questão, uma vez que foram dois dos Estados nacionais da região em que a DIS interveio e nos quais os vínculos regionais e transnacionais entre o pessoal especialista em saúde pública foram muito fortes. ${ }^{3}$ Retomando as ideias de Henri Pirenne e Marc Bloch com relação às vantagens de se comparar sociedades próximas, estes afirmam que, a partir desta abordagem, podemos, por exemplo, analisar se existiram influências mútuas e questionar falsas causas locais, o que permitirá identificar, em sua complexidade, as semelhanças e diferenças e, ao mesmo tempo, formular novas perguntas e problemas para pesquisas mais específicas (Pirenne e Bloch em D’ Assunção Barros, 2007). ${ }^{4}$ A partir da análise de documentação produzida, em especial pela Fundação, buscamos compreender como as ideias que orientaram a atuação da instituição foram implementadas nos dois países investigados.

\section{Modernizar o treinamento EM enfermagem}

Durante o transcurso do século XX, a capacitação adequada para intervir em saúde pública foi ganhando cada vez mais importncia e se constituiu em uma área de especialização que exigia saberes diferentes daqueles que se ministravam nas Escolas de Medicina. Prevenir, curar e reabilitar em grandes centros hospitalares e em campanhas sanitárias representava um desafio tanto para o pessoal médico como para a multiplicidade de agentes sanitários envolvidos nessas tarefas. O uso de técnicas e tecnologias específicas, a distribuição de bens e serviços sanitários em extensas regiões (diversas quanto a suas características geográficas), a administração e o planejamento de atividades em centros assistenciais de diferentes escalas implicavam uma formação especializada (Biernat, Ramacciotti e Rayez, 2018). Embora a organização de programas específicos de saúde pública tenha surgido na Europa no século XIX, quando se iniciaram cursos nos Estados Unidos, no século $\mathrm{XX}$, estes assumiram a dianteira, e a maioria dos líderes em saúde pública realizaram sua formação em escolas estadunidenses. Os primeiros cursos para formação em saúde pública, fundados com o apoio da FR, foram estabelecidos em Johns Hopkins, Yale, Columbia e Harvard. Estas instituições se tornaram locais de prestígio e referência para quem optava pela área como especialidade. Elizabeth Fee, ao discutir a criação, em 1916, da primeira escola de saúde pública dos Estados Unidos, a Johns Hopkins School of Hygiene and Public Health, afirma que esta instituição "as a center for training international public health officers, [it] influenced the development of public health activities around the world" (Fee, 1987, p. 33).

Já Marcos Cueto e Steven Palmer, ao analisarem especificamente a organização de escolas de enfermagem nas primeiras décadas do século XX, afirmam que:

(...) elas desafiaram a abordagem tradicional da enfermagem em hospitais que, em sua maioria, eram administrados pela Igreja, e onde as mulheres religiosas, com alguma formação especial, preenchiam as principais divisões e serviços, sendo o pessoal comum da enfermagem constituído de homens e mulheres das classes populares, sem nenhuma formação (Cueto y Palmer, 2016, p. 138).

A Escola de Enfermagem de Toronto foi, segundo palavras de John Farley, "a luz mais brilhante" quanto à capacitação nessa especialidade. Esta metáfora alude, ironicamente, a uma espécie de competição entre a escola inglesa, impulsionada por Florence Nightingale, ${ }^{5}$ no final do século XIX, a quem se denominou "a dama da lâmpada", e a Escola de Toronto, chamada de "a luz mais brilhante". A Escola de Enfermagem da Universidade de Toronto, criada no início da década de 1930 com recursos da Fundação, recebeu 39\% de todas as bolsistas de enfermagem internacional da FR, mais do que qualquer outro programa nesta área (Farley, 2004, p. 230). ${ }^{6}$ Os indivíduos indicados às bolsas deveriam se submeter a um sistema competitivo de exames e de entrevistas, que procuravam medir o potencial acadêmico e as habilidades de liderança. Esta universidade acolheu um número significativo de estudantes e contou com uma quota para a admissão de afro-americanas que provinham dos Estados Unidos. É muito provável que estas estudantes tivessem menos oportunidades educativas nos Estados Unidos, de modo que esta universidade foi, para elas, um 
lugar para o estabelecimento de redes sociais e profissionais, mas dentro de certos limites estabelecidos por questões étnicas. Embora pudessem cursar disciplinas, as práticas profissionais não podiam ser realizadas no Hospital Geral de Toronto, sendo as alunas enviadas para Detroit, cidade industrial com alta porcentagem de população afro-americana (Farley, 2004).

Ainda que, para a discursividade da FR, a existência desta escola tenha permitido a capacitação e o treinamento de mulheres que, de outra forma, não teriam podido realizá-lo, isso não impediu a reprodução de diferenças e preconceitos em torno das limitaçóes que supostamente impunham a etnicidade e o lugar de origem.

A possibilidade de treinamento no exterior a partir da concessão de bolsas de estudos foi um aspecto importante da dimensão científica da atuação da Rockefeller. A Fundação teve, no plano mundial, uma atuação pioneira na concessão de bolsas de estudos para a ciência médica e a saúde pública (Faria e Costa, 2006; Faria, 2007). Para Wickliffe Rose, Diretor da DIS entre 1913 e 1923, a educação profissional era um componente essencial para o avanço da saúde pública em âmbito internacional. Na concepção de Rose, um seleto grupo de especialistas em saúde pública difundiria o conhecimento adquirido em seus respectivos países através da pesquisa, da administração e do ensino. Na interpretação de Anne-Emanuelle Birn (2006), esta abordagem não apenas economizaria recursos financeiros, permitindo que a Fundação implementasse programas em um número maior de lugares, como também os bolsistas internacionais estariam em melhor posição com relação aos representantes da Fundação Rockefeller para disseminar, de maneira relevante, teorias, práticas e valores para pesquisadores, agências governamentais, comunidades profissionais e o público em geral. Entre as "eventuais" vantagens da distribuição de bolsas de estudos estariam o desenvolvimento de relações com líderes e futuros líderes de outros países e o intercâmbio científico internacional (Birn, 2006, p. 197).

Através da concessão de bolsas de estudos, a FR objetivava a formação de pessoal para atuar em posições estratégicas em agências de saúde oficiais ou como diretores e/ou professores em escolas de higiene, saúde pública e enfermagem. Através da ocupação de cargos de chefia em instituições e departamentos governamentais em seus países de origem, os bolsistas podiam determinar orientaçóes institucionais e prioridades, refletindo algumas das ideias e práticas com as quais haviam se familiarizado durante o período de estudos. Neste sentido, como bem destaca Birn, os bolsistas eram profissionais transnacionais, movendo ideias e práticas através das fronteiras. A influência direta ou indireta dos bolsistas poderia ser sentida por muitas décadas, pois estes, além de orientarem o trabalho em instituições e/ou departamentos de saúde, eram também, muitas vezes, professores em seus países, influenciando, desta maneira, outras gerações de funcionários da saúde pública (Birn, 2006). Através dos bolsistas, a Fundação e, no caso específico de nosso estudo, a DIS, poderia ter um efeito duradouro na teoria e na prática de saúde pública nos diversos países e regiões em que atuara.

Em documento de junho de 1924, intitulado Information Concerning Fellowships Awarded by the International Health Board of the Rockefeller Foundation (em adelante, Information Concerning), o escritório da Fundação em Nova York estabelecia que:

Fellowships granted by the International Health Board are designed to meet definite needs in public health service. They are granted only to persons carefully selected for the work that needs to be done and with the understanding that the persons receiving them will, on the completion of their training, be appointed to important positions in the official health services of their own country or in schools of hygiene (Information Concerning, 1924, p. 1).

A FR objetivava, como já apontamos, a formação de pessoal para atuar, em seus países de origem, em posições estratégicas em agências de saúde oficiais ou como diretores e/ou professores em escolas de higiene, saúde pública e enfermagem. ${ }^{7}$ Entre as exigências para concorrer a uma bolsa estavam alta qualificação profissional e científica, bem como confiabilidade e qualidades de liderança. As bolsas seriam distribuídas, preferencialmente, a candidatos com menos de 35 anos. A seleção preliminar era realizada pelos 
representantes locais da FR, em acordo com as autoridades oficiais de saúde. As inscrições e a documentação exigida eram, então, enviadas ao escritório em Nova York para a decisão final. A documentação incluía histórico pessoal em formulário a ser fornecido pela Fundação, exame médico realizado por profissional confiável, ${ }^{8}$ uma declaração de cursos especiais em medicina, ciências e saúde pública realizados pelo candidato, uma lista de suas publicações sobre temas médicos e científicos, uma declaração de sua experiência profissional desde o doutoramento, bem como uma declaração de assuntos que o candidato desejava estudar a partir da bolsa. Ou seja, o candidato já deveria ter concluído a educação formal. As bolsas podiam ter duração variada, não ultrapassando um ano. Prorrogações e extensões estavam condicionadas a um histórico satisfatório e recomendações dos responsáveis pela indicação. A Fundação se reservava o direito de cancelar a bolsa em caso de conduta considerada inadequada ou prejudicial aos objetivos da Fundação (Information Concerning, 1924).

Passemos, então, a analisar a atuação da Fundação Rockefeller na formação de profissionais de enfermagem no Brasil e na Argentina. Como veremos, se as ideias que orientaram esta atuação foram similares, o desenvolvimento das atividades e os resultados restaram diferentes, variando de país para país.

\section{A FR e a enfermagem brasileira: a criação da Escola de Enfermagem AnNa Nery}

O Brasil recebeu apoio técnico e financeiro da DIS na formação de quadros através de instituições, como a Escola de Enfermagem Anna Nery - EEAN, e se beneficiou de um elevado número de bolsas de estudos. Dezenas de países contaram com a cooperação da Fundação Rockefeller, mas, segundo autores como Lina Faria, "o Brasil foi o país do continente americano no qual a Rockefeller investiu maior soma de capitais (Faria, 2007, p. 18). A análise das informações contidas em um diretório de bolsistas da FR entre 1917 e 1971 (The Rockefeller Foundation, 1972) indica, porém, que o número de bolsas concedidas pela DIS ao Brasil foi significativo não apenas no contexto latino-americano, mas no contexto geral: do total de 2.056 bolsas distribuídas pela DIS a profissionais de mais de 80 países entre 1917 e 1951, 92 bolsas (4,4\%) foram concedidas a profissionais que atuavam no país (Korndorfer, 2013). A Argentina, país também analisado neste texto, recebeu 09 (0,4\%) bolsas (Korndorfer, 2013).

Luiz Antonio de Castro Santos e Lina Faria afirmam que, se houve efeitos positivos e duradouros da ação da FR no Brasil, estes efeitos se concentraram, principalmente, "no assentamento das raízes da educação médica e das profissões de saúde". Segundo os autores, "as origens da profissionalização médica, sanitarista e da enfermagem em saúde pública [...] estão fortemente associadas aos trabalhos desta instituição no Brasil" (Castro Santos e Faria, 2004, p. 124).

Segundo Marcos Cueto e Steven Palmer, a criação da Escola de Enfermagem Anna Nery, ${ }^{9}$ no Rio de Janeiro, foi "um resultado dos esforços conjuntos da Fundação Rockefeller e das autoridades locais" (Cueto e Palmer, 2016, p. 136). Em 1921, Carlos Chagas, então diretor do Departamento Nacional de Saúde Pública (DNSP), solicitou a cooperação da DIS para o desenvolvimento de um serviço de enfermagem em conexão com o Departamento. Aceito o convite, foi enviada ao Brasil a enfermeira Ethel Parsons, chefe da Missão Técnica de Cooperação para o Desenvolvimento da Enfermagem. A "Missão Parsons”, como ficou conhecida, tinha como objetivo realizar a avaliação das condições existentes para a organização de uma escola ou cursos de treinamento, bem como para o desenvolvimento de serviço público de enfermagem no Brasil. O projeto da Missão visava "à criação de uma sólida base para a introdução de uma nova categoria profissional no campo da saúde no Brasil" (Barreira, Baptista, Sauthier, Santos, Peres, Oliveira e Almeida Filho -em diante, Barreira et al-2015, p. 233-234). Havia a necessidade, segundo Martha Cristina Nunes Moreira (1999), de se ultrapassar uma história anterior, na qual as ações de cuidado se encontravam espalhadas pelo tecido social, sem controle.

Entre o final de 1921 e o início de 1922, Ethel Parsons analisou a situação da enfermagem no país. Em relatório, a enfermeira norte-americana afirmou: "There are no trained nurses in Brasil. A few sporadic 
attempts have been made to start training schools, but without results" (National Department of Public Health, 1922, s/p). Ao analisar as manifestações de Parsons sobre a enfermagem no país, Moreira afirma que:

Todo o esforço de observação da enfermeira norte-americana é no sentido de enfatizar a precariedade das práticas de enfermagem no Brasil, se comparadas com os padrões anglo-saxônicos, e a urgência de revê-las e profissionalizá-las, introduzindo-se coeficientes que as distinguissem perante o público médico e o leigo dos padrões até então vigentes. Os rituais de seleção deveriam englobar critérios de classe, gênero e moralidade destinados a fabricar os novos emblemas da profissão (Moreira 1999, s/p).

Em 1923, Chagas criou o Serviço de Enfermeiras, sob a direção de Parsons, assistida por outras sete enfermeiras norte-americanas. Também em 1923, o Departamento Nacional de Saúde Pública criou, com recursos da FR, uma escola de enfermagem ${ }^{10} \mathrm{em}$ instalações anexas ao Hospital Geral São Francisco de Assis, no Rio de Janeiro. A criação da escola foi oficializada pelo Decreto 16.300, de dezembro daquele ano, e que determinava que o Serviço de Enfermeiras do DNSP teria a seu cargo uma escola para instrui-las e diplomálas. O objetivo da escola era educar enfermeiras profissionais para serviços sanitários e trabalhos gerais ou especializados de hospitais e clínicas privadas.

Os rituais de admissão e formação da Escola se dariam sob o regime de internato e o curso, com duração de dois anos e quatro meses (posteriormente ampliado para três anos), possuía um currículo espelhado no modelo norte-americano. Apostilas traduzidas eram utilizadas como material didático, em atividades ministradas por médicos brasileiros e enfermeiras dos Estados Unidos (Moreira, 1999, s.p.).

Com relação ao perfil das alunas, alguns estudos afirmam que as moças deveriam ser solteiras, de boa aparência, instruídas, de modos educados e capazes de obter cartas de recomendação de homens bem colocados (Barreira et al., 2015). Segundo Moreira (1999, s/p), o diploma da Escola Normal funcionaria como um "pré-requisito", triando social e culturalmente as candidatas à Escola. Havia, ainda, um critério não formalizado, ser branca: "tentou-se assim barrar o acesso à profissão não apenas às mulheres originárias das classes menos favorecidas, como àquelas oriundas do contingente populacional majoritário de negros e mestiços" (Moreira, 1999, s/p).

A primeira turma da Escola de Enfermeiras do DNSP, com 15 alunas, formou-se em 1925. No ano seguinte, em 1926, a Escola passou a se chamar Escola de Enfermeiras D. Anna Nery. Naquele mesmo ano, em virtude do crescimento da instituição, foram inauguradas novas instalações, localizadas próximas ao Hospital São Francisco de Assis, construídas com apoio da FR.

Em 1931, com a regulamentação do ensino da enfermagem no Brasil (Decreto 20.109, de 15 de junho), que fixou as condições para a equiparação das escolas do país, o título de enfermeira ou enfermeiro diplomado só era reconhecido depois de registrado no DNSP, e a Escola de Enfermeiras D. Anna Nery, subordinada ao Departamento, foi considerada a escola oficial padrão.

Em 1937, a então Escola de Enfermagem Anna Nery foi incorporada, como instituição complementar, à Universidade do Brasil. Em 1965, passou a denominar-se Escola de Enfermagem Anna Nery da Universidade Federal do Rio de Janeiro - UFRJ. ${ }^{11}$

Entre 1917 e 1951, 88 pesquisadores e profissionais ligados a instituições e/ou departamentos governamentais brasileiros receberam 92 bolsas da DIS da Fundação Rockefeller para realizar estudos, no Brasil e no exterior, nas áreas de saúde pública ( 49 bolsas), enfermagem (31 bolsas), medicina (08 bolsas) e ciências biológicas ( 04 bolsas). Das 31 bolsas concedidas a pesquisadores e profissionais que atuavam no Brasil para que realizassem cursos na área da enfermagem, todas foram concedidas a mulheres. Receberam bolsas da DIS/FR para estudar enfermagem Glete de Alcântara, Célia Peixoto Alves, Zulema Lima Castro Amado, Luiza Antônia de Barros Thenn Araújo, Iracema dos Santos Cabral, Agnes Stuart Waddell Chagas, Aurora Gipsofila de Afonso Costa, Haydee Guanais Dourado, Edith Magalhães Fraenkel, Zilda Almeida Carvalho Hughes, Lúcia Jardim, Hilda Anna Krisch, Olga Campos Salinas Lacorte, Josette Marie Ledoux, ${ }^{12}$ Yolanda Lindenberg Lima, Rachel Haddock Lobo, Alaíde Duffles Teixeira Lott, Sylvia Arcoverde de Albuquerque 
Maranhão, Iracema dos Guaranys Mello, Delizeth de Oliveira Cabral Miyoshi, Maria de Castro Pamphiro, Alayde Borges Carneiro Paraense, Radcliff Dourado dos Santos Pereira, Maria Rosa Sousa Pinheiro, Maria do Carmo Ribeiro Prado, Maria de Oliveira Regis, Laís Moura Netto dos Reys e Zaíra Cintra Vidal (The Rockefeller Foundation, 1972). Apenas em 1948, praticamente no final do período aqui analisado, Mary Barhyte Waddell recebeu uma bolsa da DIS para estudar saúde pública nos Estados Unidos. ${ }^{13}$

As 28 brasileiras que receberam bolsas da DIS para realizar estudos na área da enfermagem não eram casadas, acompanhando o ideal presente na formação de enfermeiras em que se sustentava que as mulheres solteiras ou viúvas eram as que estariam em melhores condições de exercer a enfermagem, pois possuíam certos "dotes" biológicos para o cuidado com o outro (Ramacciotti e Valobra, 2010).

Várias bolsistas (21) haviam estudado na EEAN e 13 possuíam vínculo com a instituição quando foram contempladas com a bolsa. Já indicamos que a Fundação participou da criação e organização da Escola. Segundo Barreira et al., havia, desde 1925, ano de formatura da primeira turma da Anna Nery, um projeto de formação de uma "elite nativa”, “(...) que pudesse garantir uma inserção vantajosa da enfermagem na sociedade brasileira e, também, viesse a manter uma interlocução privilegiada com as líderes da enfermagem norte-americanas" (Barreira et al., 2015, p. 238). Como parte deste projeto, as melhores alunas de cada classe recebiam bolsas para cursos nos Estados Unidos, em diferentes campos de atuação da enfermagem, como saúde pública, administração hospitalar, pedagogia, organização de escolas de enfermagem, psiquiatria e pronto-socorro.

A primeira bolsista brasileira de enfermagem da DIS foi Edith Magalhães Fraenkel. Neta de Benjamin Constant, formada pela Escola de Enfermagem da Cruz Vermelha, Fraenkel estudou enfermagem no Philadelphia General Hospital entre 1922 e 1925. Ao retornar ao país, tornou-se instrutora e coordenadora de ensino na Anna Nery (Secaf e Costa, 2010). O objetivo de formar pessoal para assumir posiçóes de liderança nos países de origem foi sublinhada em relatório de 1926 sobre o desenvolvimento da enfermagem no Brasil: "It is understood that on the completion of her course she [Edith Fraenkel] would return to Brazil to assume a position of leadership in the Service of Nursing" (Rockefeller Foundation, 1921-1926, p. 6).

Apesar do destaque conferido à Escola de Enfermagem da Universidade de Toronto quando se discute a formação de enfermeiras, a maioria das bolsistas brasileiras de enfermagem da DIS realizou seus estudos nos Estados Unidos, mais especificamente no Philadelphia General Hospital, que recebeu 15 bolsistas. Sete das 28 bolsistas foram enviadas para a University of Toronto School of Nursing, no Canadá. É importante sublinhar que os bolsistas da Fundação não ficavam restritos à instituição onde realizavam sua formação, mas visitavam escolas, universidades, associaçóes e departamentos governamentais durante o período de estudos.

Destacaremos, a seguir, alguns aspectos da trajetória de uma das bolsistas de enfermagem, Glete de Alcântara, que foi uma das mulheres latino-americanas a realizar estudos de pós-graduação em enfermagem em saúde pública na Escola de Enfermagem da Universidade de Toronto. Glete de Alcântara nasceu em São Sebastião do Paraíso, Minas Gerais, em 24 de junho de 1910. Formada pela Escola Normal e tendo realizado também o curso de formação de educadoras sanitárias do Instituto de Higiene de São Paulo, Glete de Alcântara atuava como educadora sanitária na Prefeitura de São Paulo quando foi selecionada, juntamente com Maria Rosa Sousa Pinheiro, Zilda Almeida Carvalho Hughes e Lúcia Jardim, para estudar na Escola de Enfermagem da Universidade de Toronto. A bolsa foi concedida como parte das tratativas entre a FR e São Paulo para a criação de uma escola de enfermagem. Glete de Alcântara diplomou-se pela Universidade de Toronto em 1944. Ao retornar ao Brasil, passou a lecionar Arte da Enfermagem na então denominada Escola de Enfermagem de São Paulo. Em 1947, iniciou o curso de Ciência Sociais na Universidade de São Paulo - USP, mas suspendeu os estudos em 1950, pois recebeu uma bolsa da Fundação Kellogg para cursar pósgraduação no Teacher's College (Universidade de Colúmbia), onde obteve o título de Mestre. Ao retornar ao país, em 1951, formou-se em Ciências Sociais. Fundou, em 1953, a Escola de Enfermagem de Riberão Preto, onde, após 10 anos, conseguiu, através de concurso, o título de Professor Catedrático de História da Enfermagem e Ética, tornando-se a primeira Professora Catedrática de Enfermagem da América Latina. 
Dirigiu a Escola até 1971, vindo a falecer alguns anos depois, em 1974. Além de atuar na direção da Escola e na docência, Glete de Alcântara realizou muitas outras atividades. Foi, por duas vezes, Presidente da Associação Brasileira de Enfermagem (1952-1954 e 1972-1974), atuou como membro da Comissão de Peritos de Enfermagem da OMS (1966-1974), do Conselho Universitário da USP (1964-1971) e como Consultora Pan-americana de Saúde (México, 1967-1969). Organizou e participou de diversos eventos relacionados à enfermagem, bem como publicou uma série de trabalhos na área. ${ }^{14}$

Como podemos perceber, Glete de Alcântara teve atuação significativa na enfermagem brasileira, assim como Edith Fraenkel, Laís Moura Netto dos Reys, Maria Rosa Sousa Pinheiro, Haydée Guanais Dourado e Rachel Haddock Lobo, por exemplo, cujos nomes aparecem inúmeras vezes em levantamento realizado na Revista Brasileira de Enfermagem, seja organizando e participando de eventos e associações, seja publicando artigos.

Apesar de alguns percalços, como as críticas ao critério étnico não formalizado para ingresso na Escola, a experiência da FR na enfermagem brasileira, a partir da organização da Anna Nery, foi considerada um sucesso (Farley, 2004). Já no final dos anos 1920, porém, a perspectiva da Fundação com relação à formação em enfermagem mudaria: as escolas para formação na área deveriam estar vinculadas a universidades, e não mais a hospitais. Assim, a partir de então, a instituição organizada com recursos da FR no Rio de Janeiro "was an antiquated model unsuitable for the training of a new cadre of nursing professionals" (Farley, 2004, p. 224). Esta nova perspectiva se faria presente nos projetos da Fundação para a formação de enfermeiras na Argentina, já na década de 1940.

\section{Um Cenário Politicamente instável: A FR e a enfermagem na Argentina}

Na Argentina, em 1941, a FR tentou levar a cabo uma experiência de capacitação sanitária e de enfermagem em saúde pública com a colaboração das autoridades locais, sanitárias e universitárias. Um dos eixos foi o estímulo à Escola de Enfermagem do Hospital do Centenário de Rosário, pertencente à Universidade Nacional do Litoral. Esta escola funcionava desde junho de 1940 e foi criada por decisão da universidade no ano anterior. Para iniciar as atividades, contratou-se uma enfermeira norte-americana, Jean Martin White. ${ }^{15}$ A iniciativa contou com o apoio da Província e de médicos locais como David Sevlever, da Cadeira de Higiene, o Decano Dr. David Staffieri e o presidente da Comissão de Hospitais e Assistência Social de Santa Fé. A oferta curricular estava vinculada à Associação de Enfermagem Americana. O apoio do governo local, a cargo do Dr. Abelardo Irigoyen Freyre, e o financiamento da FR constituíram o pontapé inicial para tal empreendimento (White, 1941).

Lewis Wendell Hackett ${ }^{16}$ analisou de maneira entusiasmada as reformas sociais e sanitárias que se realizaram na província de Santa Fe Ali se instituiu o primeiro Ministério de Saúde e Trabalho na Argentina, sob a direção de seu ministro, o Dr. Abelardo Irigoyen Freyre, que se mostrou disposto a trocar ideias com o Diretor Regional (Hackett, 1941) estimulou a reforma de planos de estudo, com a inclusão de matérias ligadas às ciências sociais e à medicina preventiva, e buscou oferecer à província um organograma coordenado para a atenção sanitária. Este contexto político local foi considerado favorável para o desenvolvimento de programas sanitários auspiciados pela FR. Como consequência, a DIS facilitou a liberação de recursos para o pagamento de salários e a compra de equipamento para a criação da Escola de Enfermagem na Universidade Nacional do Litoral em Rosário.

A DIS ofereceu, ainda, recurso adicional para melhorar o salário do pessoal docente, composto por renomadas enfermeiras especializadas em saúde pública como a uruguaia Alicia Galland, ${ }^{17}$ Gwendolyn Foster, Lisette Gorsten, Sofia Andrade e Esther Hirst. ${ }^{18}$ Como contrapartida, o governo de Santa Fé ofereceu dez bolsas para as estudantes que estivessem dispostas a trabalhar em saúde pública depois de sua graduação (White, 1941, p. 11). Tal como aponta Ana Laura Martin, a avaliação que as enfermeiras norte-americanas 
realizaram sobre a situação da enfermagem na região era alarmante. $O$ treinamento necessitava de um "dramático ajuste e modificação de seus programas" e "um salto de 50 anos para frente". A enfermagem na Argentina se encontrava em condições análogas às da enfermagem britânica e norte-americana antes da segunda metade do século XIX. Esta avaliação, como é possível perceber, era bastante semelhante à realizada pela Missão Parsons no que se referia à situação da enfermagem no Brasil na década de 1920. O ensino da enfermagem em Buenos Aires dependia de instituições e de hospitais que acomodavam cursos e escolas. A formação na área não possuía status universitário e apresentava um esquema heterogêneo e pouco coordenado em matéria de conhecimentos e instrução (Martin, 2017).

No momento em que a FR concedeu a ajuda, a escola contava com dez alunas. Depois de estabelecido o acordo, matricularam-se 32 jovens, das quais 22 concluíram a capacitação. Estipulou-se, com as autoridades locais, que as enfermeiras, quando terminassem sua preparação, poderiam trabalhar nos hospitais e centros de saúde. Este incremento de cerca de 120\% no número de formadas, embora não cobrisse as necessidades existentes na região nem atendesse os ideais pautados, foi analisado pela FR como uma conquista muito significativa para a região. Segundo os informes da FR, o incremento de formadas e seu trabalho em hospitais constituiriam um estímulo para que outras jovens vissem a enfermagem como uma "profissão aberta às mulheres na Argentina" ou "um novo campo de atividades para a juventude feminina do país". ${ }^{19}$

Os requisitos para a admissão à escola universitária eram semelhantes aos do Brasil, ou seja, secundário completo e a preferência por mulheres que proviessem das escolas normais e fossem solteiras ou viúvas.

$\mathrm{Na}$ Argentina, a Revolução de Junho de $1943 \mathrm{fez} \mathrm{com} \mathrm{que} \mathrm{as} \mathrm{autoridades} \mathrm{militares} \mathrm{que} \mathrm{assumiram} \mathrm{o}$ governo interrompessem as atividades da FR. ${ }^{20}$ A partir das eleições de fevereiro de 1946 e da chegada ao poder do presidente Juan Domingo Perón, relações com a FR foram descontinuadas e tampouco foram incentivados projetos sanitários sob seu auspício (Hackett, 1946).

As referências de Hackett, em seus Diários, às reformas sanitárias levadas a cabo na Argentina, são interessantes. Por um lado, mostrou-se muito cioso delas, uma vez que se vinculavam às propostas que a FR vinha apregoando já há várias décadas. Dentre algumas das políticas implementadas durante o governo peronista, podemos destacar a construção de centros de saúde e hospitais, a realização de campanhas sanitárias contra endemias e epidemias e a criação de espaços formativos para o pessoal sanitário, tanto para médicos sanitários como para enfermeiras (Ramacciotti e Valobra, 2017). Por outro lado, Hackett foi muito crítico quanto à preeminência que as preferências políticas assumiam na designação de cargos, em detrimento das habilidades técnicas. Ao avaliar a criação da Escola de Enfermeiras de Saúde Pública, criada pela Secretaria de Saúde em meados de 1947, foi muito pessimista em relação a seu futuro, embora ele tenha advogado em favor da importância de melhorar a formação das enfermeiras. Sustentou, sem maiores detalhes, que a preparação era insuficiente, que apenas 15 jovens haviam se inscrito e que somente 12 estavam frequentando o curso em meados de 1947. Além disso, segundo Hackett, a biblioteca não contava com livros para sua capacitação e não havia um sistema de internato. As críticas mais agudas eram dirigidas à Escola de Enfermeiras da Fundação Eva Perón, visto que admitia mulheres com estudos primários. É provável que a carência de vínculos entre estas escolas de enfermagem e o âmbito universitário tenha sido um elemento que reforçou a postura crítica de Hackett. Para a FR, as escolas de enfermagem deveriam manter relações com os espaços de educação superior. Em suas intervenções na região, a instituição norte-americana potencializou essas relações, como ocorreu no caso chileno ou na experiência implementada em Santa Fe.

Um caso que merece ser destacado, pois dá conta da instabilidade política entre os EUA e a Argentina e como estas relações afetaram os vínculos entre a FR e a Argentina, foi o da enfermeira María Elena Ramos Mejía. Ramos Mejía foi diretora da Escola Municipal “Cecilia Grierson” durante a década de 1940, local em que se formaram cerca de quatrocentas enfermeiras e que era conceituado como um dos espaços de maior prestígio em Buenos Aires. Ramos Mejía solicitou uma bolsa para capacitar-se nos EUA em 1942. Sua solicitação cumpria todos os requisitos exigidos, e, nos informes da FR, destacavam-se tanto sua vinculação 
com uma tradicional família de médicos e políticos da Argentina quanto seu perfil internacional, uma vez que tinha participado de congressos na Europa.

Considerando que a FR identificava, na Argentina, a inexistência de vínculos entre as escolas de enfermagem e a universidade como um dos principais problemas, acreditava-se que conceder uma bolsa a Ramos Mejía seria uma oportunidade para que ela pudesse conhecer o padrão formativo em outras latitudes, obter capacitação no Canadá e depois divulgar essa experiência em Buenos Aires.

Durante o transcurso do ano de 1942, Ramos Mejía cumpriu com todos os requisitos para que seu pedido fosse levado à FR: apresentou informações sobre sua formação, planilhas com dados pessoais e as recomendações de Hackett e Mary Elizabeth Tennant, integrante da equipe técnica da DIS na área de enfermagem e saúde pública. Ramos Mejía obteve a bolsa por um período de oito meses, tempo de que dispunha para estar fora do país por razões pessoais e laborais.

Contudo, o visto para ingressar nos Estados Unidos lhe foi negado. As autoridades dos EUA receberam uma denúncia anônima sobre supostas relações e amizades com os círculos locais alemães. Em um contexto em que a Argentina mantinha política de neutralidade na Segunda Guerra Mundial (1939-1945), estes aparentes vínculos foram interpretados pelos EUA como um sinal negativo e acabaram sendo determinantes para que seu ingresso no país fosse negado. Ramos Mejía, em carta dirigida à FR, considerou esta explicação ridícula, pois, embora tivesse admiração pela disciplina e cultura alemãs, isso não era um indicador de suas simpatias pelo nazismo. Além disso, apelava para os laços familiares com sua família de origem inglesa como garantia de seu apego aos valores democráticos. Alegava que seu apelido de "a alemã” se devia ao seu apreço pela disciplina e pelo respeito à autoridade. Afirmava, ainda, que o apelido remetia à sua maneira de organizar a escola e não podia ser associado a questóes políticas.

Apesar de sua carta, a negativa foi mantida e ela teve que renunciar à bolsa que lhe havia sido concedida. Ramos Mejía afirmou esperar que, em outro momento, quando "as paixões políticas estivessem mais adormecidas”, ela pudesse realizar esta capacitação (Ramos Mejía, 1942, s/p).

Hackett tentou reverter a medida tomada pelas autoridades, mas fracassou em sua intermediação. Em seus diários, sustentou que era uma ação injusta e que se sentia envergonhado pela decisão tomada, já que considerava que não existia outra pessoa na Argentina que pudesse organizar uma escola de enfermagem universitária. No entanto, tentava justificar a medida indicando que, em tempos de guerra, "há injustiças mais sérias”. Em 1946, Hackett seguia lamentando que Ramos Mejía não tivesse podido viajar aos EUA ou Canadá para aprimorar sua formação (Hackett, 1946). Um ano mais tarde, em 1947, Ramos Mejía abandonou suas atividades profissionais, pois contraiu matrimônio. Contudo, antes de retirar-se, teve um papel destacado na Comissão de Cultura Sanitária, organizada pelas autoridades sanitárias do peronismo para realizar a reforma dos planos de estudo de enfermagem (Martin, 2017).

\section{CONSIDERAÇões FINAIS}

Neste trabalho, propusemo-nos a discutir a atuação da DIS/FR na formação de enfermeiras no Brasil e na Argentina, sublinhando semelhanças e diferenças. $\mathrm{O}$ modelo formativo foi semelhante para toda a região. A profissionalização de enfermeiras teve como modelo aquele desenvolvido nos Estados Unidos e no Canadá. O objetivo era difundir noções de prevenção sanitária a partir da atuação das enfermeiras capacitadas, instruídas nos modernos princípios da enfermagem.

A escolha do Brasil e da Argentina para esta análise se deve ao fato de terem sido países em que a FR atuou, na América, na primeira metade do século XX. Nos dois países, as atividades da DIS ocorreram em momentos diferentes e tiveram resultados bastante distintos. Embora as experiências tenham sido promovidas com os mesmos objetivos - capacitação (vinculada a espaços universitários, no caso e argentino), disseminação de conteúdos relacionados à medicina preventiva, treinamento de mulheres com curso secundário completo e de setores médios, práticas ligadas à saúde pública -, os resultados foram bem díspares. 
O caso brasileiro, desenvolvido a partir do período entreguerras, foi o mais destacado dada a quantidade de bolsas concedidas e os estreitos vínculos mantidos entre a FR e a Escola Anna Nery, considerada umas das mais importantes da região em termos de enfermagem sanitária. A maioria das 28 bolsistas brasileiras de enfermagem da DIS realizaram seus estudos nos Estados Unidos. Ao retornarem, várias acabaram por ocupar postos importantes no desenvolvimento da enfermagem no Brasil, como foi o caso de Glete de Alcantara. Mesmo que, no final dos anos 1920, a perspectiva da Fundação com relação à formação em enfermagem tenha mudado -as escolas para formação na área deveriam estar vinculadas a universidades, e não mais a hospitaisquando de sua organização, a EEAN foi considerada um sucesso.

A experiência argentina, fomentada nos anos quarenta, por sua vez, durou somente três anos e foi cancelado em virtude da interrupção da vida democrática. Embora, na Argentina, se vissem encerrados os projetos de intercâmbio entre as autoridades locais e a FR, as ideias, as redes de contatos e a influência regional dos mencionados modelos formativos continuaram operando nas políticas de reforma curricular da enfermagem propostas pela Secretaria de Saúde Pública e pela Fundação Eva Perón.

A ditadura militar de 1943 interrompeu os programas da FR. Depois do golpe de Estado de 1943, a equipe docente e administrativa da Escola de Enfermagem de Rosário foi suspensa por decreto e os contratos não foram renovados. A escola deixou de funcionar em 1945. A política se inseriu na vida universitária, e a confrontação e a instabilidade institucional se polarizaram. Um espaço formativo ligado à capacitação técnica de enfermeiras foi envolvido pelas posturas nacionalistas da época, que apontavam a influência norteamericana como sinal de intervenção na vida política local. De outro ponto de vista, a postura de neutralidade da Argentina diante da Segunda Guerra Mundial e quaisquer indícios de inclinação ao Eixo foram elementos apontados como justificativas para o rompimento de relação entre os Estados Unidos e a Argentina. Neste sentido, o visto negado a Ramos Mejía para ingresso nos EUA é um exemplo disso. Com o governo de Perón, organizou-se uma Comissão de Cultura Sanitária, criada pela Secretaria de Saúde Pública, que teve como função unificar diplomas e programas de estudos para capacitar enfermeiras nas tarefas assistenciais e nas vinculadas à saúde pública de massas. Apesar destas tentativas de modernizar o treinamento, na Argentina, a vinculação entre a capacitação das enfermeiras e a vida universitária teve que esperar até 1952, momento em que a Universidade Nacional de Tucumán estabeleceu a Escola de Enfermeiras sob sua órbita.

\section{REFERÊNCIAS DOCUMENTAIS}

Curriculum Vitae (RAC, RF, RG Special Collection - Lewis Hackett, Series 6.2 - 6.6 Biographical Information, Box 23, Folder 206).

Hackett, L. Diaries, 1941 (Rockefeller Foundation records, officers' diaries, RG 12, F-L (FA392). https ://dimes.rockarch.org.

Hackett, L. Diaries, 1946 (Rockefeller Foundation records, officers' diaries, RG 12, F-L (FA392). https ://dimes.rockarch.org.

Hackett, L. (RAC, RF, RG Special Collection - Lewis Hackett, Series 6.2 - 6.6 Biographical Information, Box 23, Folder 206).

Fellowship Cards (RAC, RF, RG 10.2).

History of the Fellowship Program at the Rockefeller Foundation (RAC, RF, RG 10.2, Fellowship Recorder Cards).

Information Concerning Fellowships Awarded by the International Health Board of the Rockefeller Foundation (Acervo Histórico da Escola de Enfermagem Anna Nery - Universidade Federal do Rio de janeiro, Centro de Documentação, Caixa 09, Doc. 57, 1924).

Hackett, L. (RAC, RF, RG Special Collection - Lewis Hackett, Series 6.2 - 6.6 Biographical Information, Box 23, Folder 206). 
National Department of Public Health (1922). Narrative Report of the Service of Nursing and Nursing Education of the National Department of Public Health - Rio de Janeiro, September 1, 1921 to April 1, 1922 (RAC, RF, RG 5.3, Series 303, Box 107, Folder 1402).

Ramos Mejía, M. (1942). RAC, RF, RG 2, Series 301, Box 236, Folder 1635.

Rockefeller Foundation Nursing Litoral (RAC, RF, RG 1. 1, Series 301, Box 4, Folder 38).

Rockefeller Foundation (1926). Confidential Report of Medical Examiner (Acervo Histórico da Escola de Enfermagem Anna Nery - Universidade Federal do Rio de Janeiro, Centro de Documentação, Caixa 09, Doc. 56).

Rockefeller Foundation (1972). Directory of Fellowships and Scholarships (1917-1971). Nova York: The Rockefeller Foundation.

Rockefeller Foundation (1921-1926). The Development of a Service of Nursing in Brazil, 1921-1926 (RAC, RF, RG 5.3, Series 305C, Box 110, Folder 1421).

White, J. (1941). University School in Argentina. The American Journal of Nursing, 41(6), 659-662.

\section{REFERÊNCIAS}

Angerami, E. e Pelá, N. (1976). Glete de Alcântara: vida e obra. São Paulo: Empresa Gráfica da dos Tribunais S.A.

Barreira, I., Baptista, S., Sauthier, J., Santos, T., Peres, M., Oliveira, A. e Almeida Filho, A. (2015). A Primeira República: a implantação da enfermagem laica e seus desdobramentos (1889-1930). In M. Padilha, M. Borenstein e I. Santos (Org). Enfermagem: história de uma profissão (pp. 225-258). São Caetano do Sul, SP: Difusão Editora.

Biernat, C., Ramacciotti, K. y Rayez, F. (2018). La capacitación en salud pública en la Argentina entre 1900-1960, História Unisinos, 22(4), 637-650.

Birn, A. (2006). Marriage of Convenience: Rockefeller International Health and revolutionary Mexico. Rochester: University of Rochester Press.

Bloch, M. (1998). Para uma história comparada das sociedades européias. In E. Bloch. História e Historiadores (pp. 119-150). Tradução de Telma Costa. Lisboa: Teorema.

Casa de Oswaldo Cruz (s/f). Escola de Enfermeiras do Departamento Nacional de Saúde Pública (pp. 1-11). Em Dicionário Histórico-Biográfico das Ciências da Saúde no Brasil, 1832-1930. Fiocruz. Recuperado de http://w ww.dichistoriasaude.coc.fiocruz.br/iah/pt/index.php

Castro Santos, L. e Faria, L. (2004). Cooperação internacional e a enfermagem de saúde pública no Rio de Janeiro e São Paulo. Horizontes, 22(2), 123-150.

Costa, R. et al. (2015). Florence Nightingale (1820-1910): as bases da enfermagem moderna no mundo. In M. Padilha, M. Borenstein e I. Santos (Org.). Enfermagem: história de uma profissão (pp. 187-224). São Caetano do Sul, SP: Difusão Editora.

Cueto, M. (1994). Missionaries of science: the Rockefeller Foundation and Latin America. Bloomington: Indiana University Press.

Cueto, M. e Palmer, S. (2016). Medicina e Saúde Pública na América Latina: uma história. Rio de Janeiro: Editora Fiocruz.

D’ Assunção Barros, J. (2007). Historia comparada. Um novo modo de ver e fazer historia. Revista de Historia Comparada, 1(1), 1-30. Recuperado de https://revistas.ufrj.br/index.php/RevistaHistoriaComparada/article/ view/144/136

Faria, L. (2007). Saúde e Politica: a Fundação Rockefeller e seus parceiros em São Paulo. Rio de Janeiro: Editora Fiocruz.

Faria, L. e Costa, M. (2006). Cooperação Científica Internacional: Estilos de Atuação da Fundação Rockefeller e da Fundação Ford. Dados - Revista de Ciências Sociais, 49(1), 159-191.

Farley, J. (2004). To cast out disease: a history of the International Health Division of the Rockefeller Foundation (1913-1951). Nova York: Oxford University Press. 
Fee, E. (1987). Disease and Discovery: a History of the Johns Hopkins School of Hygiene and Public Health, 1916-1939. Baltimore: The Johns Hopkins University Press.

Gutiérrez Urquijo, N. (2019). Las enfermeras visitadoras y la Fundación Rockefeller en Colombia a inícios de los años treinta. Rockefeller Research Report. Recuperado de http://rockarch.issuelab.org/resource/visiting-nurses-and -the-rockefeller-foundation-in-colombia-1929-1932.html

Korndorfer, A. (2013). An international problem of serious proportions: A cooperação entre a Fundação Rockefeller eo governo do estado do Rio Grande do Sul no combate à ancilostomiase e seus desdobramentos (1919-1929). Tese (Doutorado em História) - Faculdade de Filosofia e Ciências Humanas, Pontifícia Universidade Católica do Rio Grande do Sul, Porto Alegre.

Moreira, M. (1999). A Fundação Rockefeller e a construção da identidade profissional de enfermagem no Brasil na Primeira República. História, Ciências, Saúde - Manguinhos, Rio de Janeiro, 5(3), 631-645.

Martin, A. (2017). Organismos técnicos sanitarios y profesionalización. El caso de la enfermería. 1947-1955. In: J. Cordeu (ed.). Actas de las XVI Jornadas Interescuelas. Mar del Plata: Universidad Nacional de Mar del Plata. Recuperado de https://interescuelasmardelplata.wordpress.com/actas/

Ramacciotti, K. (2017). La Fundación Rockefeller y la División Internacional de Salud en el Río de la Plata y la Región Andina: ideas, concreciones y obstáculos (1941-1949). Redes, 23(45), 97-121.

Ramacciotti, K. e Valobra, A. (2010). La profesionalización de la enfermería en Argentina. Disputas políticas e institucionales durante el peronismo. Asclepio, Revista de Historia de la Medicina y de la Ciencia, 62(2), 353-374.

Ramacciotti, K. e Valobra, A. (2017). El dilema Nightingale: controversias sobre la profesionalización de la enfermería en Argentina 1949-1967. Dynamis, 37(2), 367-387.

Romero, L. (1994). Breve Historia Contemporánea de la Argentina. Buenos Aires: Fondo de Cultura Económica.

Sauthier, J. e Barreira, I. (1999). As enfermeiras norte-americanas e o ensino de enfermagem na capital do Brasil: 1921-1931. Rio de Janeiro: Editora da Escola de Anna Nery / UFRJ.

Secaf, V. e Costa, H. (2010). Enfermeiras do Brasil: história das pioneiras. São Paulo: Biblioteca 24 horas.

Vessuri, H. (2001). Enfermaría de salud pública, modernización y cooperación internacional. El proyecto de la Escuela Nacional de Enfermeras de Venezuela, 1936-1950. Rio de Janeiro, História, Ciências, Saúde - Manguinhos, 8(3), 507-539. https://doi.org/10.1590/S0104-59702001000400002

Wilkinson, L. (2000). Burgeoning Visions of Global Public Health: The Rockefeller Foundation, The London School of Hygiene and Tropical Medicine, and the "Hookworm Connection". Studies in History and Philosophy of Biological \& Biomedical Sciences, 31(3), 397-407.

\section{Notas}

1 A Divisão Internacional de Saúde da Fundação Rockefeller chamou-se International Health Commission (IHC), entre 1913 e 1916, International Health Board (IHB) entre 1916 e 1927 e International Health Division (IHD) entre 1927 e 1951 e, ao encerrar suas atividades em 1951, havia estado presente em mais de 80 países do mundo, incluindo todos os países da América do Sul (Farley, 2004).

2 Agradecemos a leitura atenta e as contribuições de Cláudio Pereira Elmir e Cristiano Enrique de Brum na construção deste texto.

3 Há estudos sobre a atuação da Fundação Rockefeller na formação de enfermeiras em outros países da América do Sul, como a Venezuela, onde ocorreu a criação, na década de 1940, da Escuela Nacional de Enfermeras (ENE), e a Colômbia, país que contou com importante presença da instituição norte-americana entre 1919 e 1945. Para maiores informações sobre a atuação da FR nos dois países e, mais especificamente, na formação de enfermeiras, consultar Vessuri, 2001; Gutiérrez Urquijo, 2019.

4 Marc Bloch escreveu, em 1928, o texto que é, ainda hoje, referência fundamental nos debates e discussões sobre a história comparada. Em Para uma história comparada das sociedades europeias, Bloch pretendeu demonstrar que o método comparativo é "um instrumento técnico de uso corrente, maleável e susceptível de resultados positivos" (Bloch, 1998, p. 120). O autor buscou, assim, precisar a natureza e as possibilidades de aplicação da "boa ferramenta" que é a comparação e, por meio de exemplos, indicar o que é possível esperar do método, além de sugerir meios práticos para facilitar seu uso. Para Bloch, porém, o método comparativo mais rico cientificamente é o mais limitado em seu horizonte: “[...] estudar 
paralelamente sociedades a um tempo vizinhas e contemporâneas, incessantemente influenciadas umas pelas outras, cujo desenvolvimento está submetido [...] à ação das mesmas grandes causas e que remontam, pelo menos em parte, a uma origem comum" (Bloch, 1998, pp. 122-123).

5 Florence Nightingale foi uma enfermeira inglesa, pioneira da enfermagem profissional moderna. O trabalho de Nightingale durante a Guerra da Criméia (1854) teria estabelecido uma melhora na organização dos serviços hospitalares baseada em estritas normas de higiene e ventilação e impulsionou mudanças na formação em enfermagem (Costa et al.,2015).

6 Segundo Cueto e Palmer (2016, p. 137), “a Faculdade de Enfermagem, da Universidade de Toronto, que, em 1943, foi a primeira escola do Canadá a oferecer uma licenciatura em enfermagem, foi a destinatária de grandes pacotes de financiamento da Fundação Rockefeller, sobretudo no seu início, quando era um pequeno departamento dentro da Escola de Saúde Pública. O programa de Toronto tornou-se um favorito para as mulheres latino-americanas que queriam fazer os estudos de pós-graduação em enfermagem em saúde pública”. Uma discussão sobre a participação da Fundação Rockefeller na organização da Toronto School of Higiene e da University of Toronto's School of Nursing pode ser encontrada em Farley, 2004, pp. 216-238.

7 Os objetivos do programa de bolsas da Fundação são expressos, também, em History of the Fellowship Program at the Rockefeller Foundation: "Thus the functions of the Rockefeller Foundation (RF) fellowship program have been to select individuals of outstanding promise in the fields of interest defined by the general program of the Foundation, and to help to prepare individuals to make significant contributions to research and teaching or public health in the future" (RAC, RF, RG 10.2, Fellowship Recorder Cards).

8 O exame médico -Rockefeller Foundation, Confidential Report of Medical Examiner- compreendia 19 itens que incluíam aspectos físicos e psicológicos (Acervo Histórico da Escola de Enfermagem Anna Nery - Universidade Federal do Rio de Janeiro, Centro de Documentação, Caixa 09, Doc. 56, 1926).

$9 \mathrm{O}$ nome conferido à Escola foi uma homenagem à pioneira enfermeira brasileira Anna Nery, que viveu entre $1814 \mathrm{e}$ 1880 e ganhou fama na Guerra do Paraguai (Cueto e Palmer, 2016, p. 136).

10 As informações sobre a Escola estão em Escola de Enfermeiras do Departamento Nacional de Saúde Pública. Em Casa de Oswaldo Cruz (s/f), Barreira et al., 2015. A atuação das enfermeiras norte-americanas no Rio de Janeiro foi discutida por Sauthier \& Barreira, 1999.

11 Outro subproduto importante da missão norte-americana, segundo Cueto e Palmer (2016, p. 136), foi a criação, em 1926, de uma associação brasileira de enfermeiras diplomadas, que se filiou, alguns anos depois, ao Conselho Internacional de Enfermeiros e começou a publicar a Revista Brasileira de Enfermagem na década de 1930. A partir dos números de 1972, as edições da Revista estão disponíveis na internet: http://www.scielo.br/scielo.php?script=sci_seri al\&pid=0034-7167\&lng=pt\&nrm=iso.

12 Josette Marie Ledoux não era brasileira (nasceu na Bélgica, segundo informações contidas em documentação da Fundação Rockefeller - RAC, RF, RG 10.2 Fellowship Cards, Series LEDOUX, Josette Marie), mas foi incluída na lista, pois é associada ao Brasil que Ledoux aparece no Diretório (1972), uma vez que sua bolsa está vinculada a atividades que desenvolveria neste país.

13 As informações referentes às bolsistas e às bolsas foram coletadas em Directory of Fellowships and Scholarships (1917-1970) (The Rockefeller Foundation, 1972), nas fichas dos bolsistas salvaguadadas no Rockefeller Archive Center (RAC, RF, RG 10.2 Fellowship Cards), em registros da Escola de Enfermagem Anna Nery (Acervo Histórico da Escola de Enfermagem Anna Nery - Universidade Federal do Rio de Janeiro, Centro de Documentação), e em publicações como Secaf e Costa, 2010.

14 As informações relativas à trajetória de Glete de Alcântara estão disponíveis em Secaf e Costa, 2010; Angerami e Pelá, 1976.

15 Jean Martin White era enfermeira diplomada pelo Kings Country Hospital de Nova Iorque. Posteriormente, obteve o bacharelado em Ciências na Universidade de Colúmbia e foi aprovada nos cursos "Nursing" da mesma universidade. Obteve diploma em cursos de higiene infantil e contava com uma vasta experiência na organização de escolas nos Estados Unidos, Chile e Havaí.

16 Hackett foi o Diretor -Associate Regional Director-da DIS para o Brasil entre 1916 e 1923. Lewis Wendell Hackett nasceu na Califórnia em 14 de dezembro de 1884. Hackett diplomou-se em Medicina pela Universidade de Harvard em 1912. No ano seguinte, em 1913, recebeu o título de Doutor em Saúde Pública, o primeiro concedido pela Universidade de Harvard, e tornou-se instrutor de Medicina Preventiva da Escola de Medicina da mesma Universidade. Em abril de 1914, Hackett foi convidado a trabalhar na DIS da Fundação Rockefeller. Entre 1940 e 1949, Hackett foi Diretor Regional para a região do Rio da Prata e dos Andes (Argentina, Bolívia, Chile, Equador, Paraguai, Peru e Uruguai). Lewis Hackett atuou em vários países durante o período em que trabalhou para a Fundação: Costa Rica, Panamá, Honduras, Guatemala, Brasil, Paraguai, Itália, Albânia, Egito, Argentina, Bolívia, Chile, Equador, Paraguai, Peru e Uruguai (Lewis Wendell Hackett - RAC, RF, RG Special Collection - Lewis Hackett, Series 6.2 - 6.6 Biographical Information, Box 
23, Folder 206; Curriculum Vitae - RAC, RF, RG Special Collection - Lewis Hackett, Series 6.2 - 6.6 Biographical Information, Box 23, Folder 206).

17 Alicia Galland se formou no Hospital de Bon Secour em Genebra.

18 Esther Hirst tinha ocupado cargos docentes na Faculdade de Medicina da Universidade de Ann Harbor em Michigan.

19 As alunas que terminaram o curso no primeiro ano foram Irene Abad, Hilda Cajal e Berteline Talpalar ("Se impuso la toca a las primeras Nurses de la Esculela de Rosario", Tribuna, Rosario, 11 de dezembro de 1940 - RAC, RF, RG 1. 1, Series 301, Box 4, Folder 37 e "La escuela de Nurses ofrece un nuevo campo de actividades a la juventud femenina del país”. Nursing Litoral, RAC, RF, RG 1. 1, Series 301, Box 4, Folder 38).

20 A Revolução de Junho de 1943 foi um golpe de Estado ocorrido na Argentina e liderado pelo General Rawson, que anunciou, em seu gabinete, integrantes ideológicamente alinhados com a Alemanha. Renunciou antes mesmo de assumir e foi substituído pelo General Ramirez, que adotou medidas contra o comunismo e os sindicatos, bem como estabeleceu uma forte aliança com grupos católicos, o que ficou evidenciado a partir do incentivo ao ensino religioso nas escolas públicas (Romero, 1994, pp. 129-131). 